\title{
Key Role of Dimensional Analysis Homogeneity in Proving
Hypothesis and Providing Explanations on the Closely Related Gram Points
}

\author{
John Y. C. Ting ${ }^{1}$ \\ ${ }^{1}$ Rural Generalist in Anesthesia and Emergency medicine, Dental and Medical Surgery, 729 Albany Creek Road, Albany \\ Creek, Queensland 4035, Australia
}

Correspondence: Dr. John Y. C. Ting, 729 Albany Creek Road, Albany Creek, Queensland 4035, Australia. E-mail: jycting@hotmail.com

Received: April 27, 2016 Accepted: June 10, 2016 Online Published: July 25, 2016

doi:10.5539/jmr.v8n4p1 URL: http://dx.doi.org/10.5539/jmr.v8n4p1

\begin{abstract}
Riemann zeta function is the famous complex number infinite series consisting of a real and an imaginary part. Nontrivial zeros and Gram points are best seen as mathematically derived entities of this function when its variable Sigma has a value of $\frac{1}{2}$. The presence [but not the actual locations] of the complete set of infinite non-trivial zeros is characterized by the criterion that the sum total of the simultaneous real and imaginary parts in Riemann zeta function equates to zero. In an identical manner this slightly altered criterion for the presence [but not the actual locations] of the complete set of infinite Gram points is that this 'sum total' now refer to the lesser requirement that only the individual imaginary part in Riemann zeta function equates to zero. The key role played by Dimensional analysis homogeneity to rigorously prove Riemann conjecture/hypothesis has been fully outlined in our landmark research paper published earlier on Page 9 - 21 in the preceding Volume 8, Number 3, June 2016 issue of this journal. Those resulting methodology previously employed by us are now mathematically used in an analogical procedure to delineate its role in successfully supplying crucial explanations for Gram points. In this research article, we use the notation \{Non-critical lines\}-Gram points to signify those 'near-identical' (virtual) Gram points when Sigma value is not $\frac{1}{2}$.
\end{abstract}

Keywords: Dimensional analysis homogeneity, Dirichlet-Gram-Riemann conjecture, Dirichlet-Gram-Riemann hypothesis, Gram conjecture, Gram hypothesis, Gram points, Riemann hypothesis

Subject classification: $11 \mathrm{M} 26,10 \mathrm{H} 05$

\section{Introduction}

Riemann zeta function is a famous example of an infinite series with complex numbers consisting of a real part and an imaginary part. The Dirichlet eta function is essentially the surrogate for Riemann zeta function. For the purpose of this study, non-trivial zeros and Gram points can best be thought of as mathematically derived entities of this function when its variable Sigma $(\sigma)$ is endowed with a value of $\frac{1}{2}$. Proposed by the famous German mathematician Bernhard Riemann in 1859, Riemann hypothesis refers to the famous conjecture explicitly equivalent to the mathematical statement that the critical line $\left(\sigma=\frac{1}{2}\right)$ in the critical strip $(0<\sigma<1)$ of Riemann zeta function is the location for all of its non-trivial zeros. Manifesting as conjugate pairs of non-trivial zeros, they can succinctly be denoted by $\zeta\left(\frac{1}{2} \pm t t\right)=0$. Gram points are the other conjugate pairs values on the critical line defined by $\operatorname{Im}\left\{\zeta\left(\frac{1}{2} \pm \imath t\right)\right\}=0$ whereby they obey Gram's Rule and Rosser's Rule with many other interesting characteristics. In practice, the positive $(0<t<+\infty)$, and numerically equal to the negative $(-\infty<t<0)$, counterpart of the conjugate pairs for the zeros of $\zeta(s)$ and its Gram points is usually quoted or employed for calculation purposes.

The $\mathrm{Z}$ function is a function used for studying the Riemann zeta function along the critical line. It is also called the Riemann-Siegel Z function, the Riemann-Siegel zeta function, the Hardy function, the Hardy Z function, and the Hardy zeta function. It can be defined in terms of the Riemann-Siegel theta function and the Riemann zeta function by $Z(t)=$ $e^{\imath \theta(t)} \zeta\left(\frac{1}{2}+t t\right)$ whereby $\theta(t)=\arg \left(\Gamma\left(\frac{(2 t t+1)}{4}\right)\right)-\frac{\log \pi}{2} t$. In this research article, unless stated otherwise, the symbol 'log' refers to natural logarithm. In the next paragraph, we will only outline a brief exposition of some of the useful properties of Gram points.

The algorithm used to compute $\mathrm{Z}(\mathrm{t})$ is called the Riemann-Siegel formula, named after Bernhard Riemann (1826 - 1866) who first discovered though he did not publish it, and C. Siegel (1896 - 1981) who finally deciphered Riemann's notes and published the result in the 1930's. The zeta function on the critical line, $\zeta\left(\frac{1}{2}+t t\right)$, will be real when $\sin (\theta(t))=0$. Positive 
real values of $t$ where this occurs are called Gram points, after J. P. Gram (1850 - 1916), and can also be described as the points where $\frac{\theta(t)}{\pi}$ is an integer. Gram observed that the real part of the zeta function on the critical line tends to be positive, while the imaginary part alternates more regularly between positive and negative values. That means that the sign of $\mathrm{Z}(\mathrm{t})$ must be opposite to that of the sine function most of the time, so one would expect the non-trivial zeros of $Z(t)$ to alternate with zeros of the sine term, i.e. when $\theta$ takes on integer multiples of $\pi$. This turns out to hold most of the time and is known as Gram's Rule (Law) - a law which is violated infinitely often though. Thus Gram's Law is the statement that non-trivial zeros of Z(t) alternate with Gram points. Gram points which satisfy Gram's Law are called 'good', while those that do not are called 'bad'. A Gram block is an interval such that its very first and last points are good Gram points and all Gram points inside this interval are bad. The exercise of counting non-trivial zeros then reduces to that of counting all Gram points where Gram's Law is satisfied, and adding to that the count of non-trivial zeros inside each Gram block. With this process we do not have to locate non-trivial zeros exactly, and we just have to compute $\mathrm{Z}(\mathrm{t})$ accurately enough to show that it changes sign.

The presence [but not the actual locations] of the complete set of infinite non-trivial zeros is characterized by the criterion that the sum total of the simultaneous real and imaginary parts in Riemann zeta function equates to zero. In an identical manner this slightly altered criterion for the presence [but not the actual locations] of the complete set of infinite Gram points is that this [non-zero] 'sum total' now refer to the lesser requirement that only the individual imaginary part in Riemann zeta function needs to be equal to zero. The key role played by Dimensional analysis homogeneity to rigorously prove Riemann conjecture/hypothesis had been formally submitted and published as a landmark research paper in the preceding Volume 8, Number 3, June 2016 issue of this journal with the title Rigorous Proof for Riemann Hypothesis using the Novel Sigma-power Laws and Concepts from the Hybrid Method of Integer Sequence Classification (Ting, 2016). Although this current article is self-sufficient, we strongly urge the interested reader to read our earlier research article for easier comprehension as the methodology previously employed by us are now used in an analogical and systematical manner to delineate its role in successfully supplying crucial explanations on Gram points. In this paper, we use the notation Non-critical lines-Gram points to signify those 'near-identical' (virtual) Gram points when $\sigma \neq \frac{1}{2}$.

\section{Gram Conjecture and Gram Hypothesis}

Gram conjecture is the mathematical postulate that all Gram points only occur on the critical line $\left[\sigma=\frac{1}{2}\right]$ in the critical strip $[0<\sigma<1]$ of Riemann zeta function when the unconditional scientific principle of Dimensional analysis homogeneity is not violated.

The main and sole proposition of this paper is outlined in the above conjecture. We stress here that to differentiate between the terms 'conjecture' and 'hypothesis' (as in the Conclusion section below), Gram conjecture can and will only be denoted by Gram hypothesis once this conjecture is mathematically proven beyond doubt in a rigorous manner towards the end of this paper.

We now maintain that the contents of this paper will provide all necessary evidence for, and are centered on, the following four theorems on Gram points as the main theme.

Theorem I: The exact same \{Modified-for-Gram points\}-Riemann-Dirichlet Ratio, directly derived from either the Riemann zeta or Dirichlet eta function, is an irrefutably accurate mathematical expression on the de novo criteria for the actual presence [but not the actual locations] of the complete set of (identical) infinite Gram points in both functions.

Theorem II: Both the near-identical (by proportionality factor-related) \{Modified-for-Gram points\}-Riemann Sigma-power law and \{Modified-for-Gram points\}-Dirichlet Sigma-power law with their derivations based on either the numerator or denominator of \{Modified-for-Gram points\}-Riemann-Dirichlet Ratio have Dimensional analysis (DA) homogeneity only when their common and unknown $\sigma$ variable has a value of $\frac{1}{2}$ as its solution.

Theorem III: The $\sigma$ variable with value of $\frac{1}{2}$ derived using the \{Modified-for-Gram points\}-Sigma-power law [from Theorem II above] is the exact same $\sigma$ variable in Gram conjecture which proposed $\sigma$ to also have the value of $\frac{1}{2}$ (representing the critical line with $\sigma=\frac{1}{2}$ in the critical strip with $0<\sigma<1$ ) for the location of all Gram points of Riemann zeta function [and Dirichlet eta function by default], thus providing irrefutable evidence for this Gram conjecture to be correct with further clarification from Theorem IV below.

Theorem IV: Condition 1. Any other values of $\sigma$ apart from the $\frac{1}{2}$ value arising from $0<\sigma<\frac{1}{2}$ and $\frac{1}{2}<\sigma<1$ in the critical strip does not contain any Gram points ["the DA-wise mathematical impossibility argument" with resulting de novo DA non-homogeneity], together with Condition 2. The one and only one value of $\frac{1}{2}$ for $\sigma$ in the critical strip contains all the Gram points ["the DA-wise one and only one mathematical possibility argument" with resulting de novo DA homogeneity] from Theorem III, fully support the rather mute, but nevertheless the whole, point of study in this paper that Gram conjecture is proven to be true when these two (mutually inclusive) conditions are met.

It is to be elaborated here that the phrase "Key role of Dimensional analysis homogeneity in proving Riemann conjecture/hypothesis or Gram conjecture/hypothesis" is contextually never intended by us as a connotation that "Laws of 
Physics along with Scientific Principles even when all of them put together fully satisfying Dimensional analysis homogeneity per se can purportedly prove mathematical theorems". Rather, of utmost significance is this Dimensional analysis homogeneity (and non-homogeneity) being the secondary consequence [in a mathematical consistent albeit seemingly indirect manner] arising naturally out of our research methods used to fully prove Theorems I - IV above. This phenomenon simultaneously occurs both in this current research paper and in our previous closely-related research paper (Ting, 2016). To also indicate continuity, the exact title of this paper, as an agenda, was briefly highlighted by us on Page 10 of the Introduction section in our earlier research paper on the complete proof for Riemann conjecture/hypothesis.

Well over 2000 years ago (c. 300 BC) Euclid proved that there were infinitely many primes predominantly by reductio ad absurdum (proof by contradiction) method. Since then dozens of proofs have been devised - three of which are chronologically presented below with the strangest candidate likely to be Furstenberg's Topological Proof.

- Goldbach's Proof using Fermat numbers (written in a letter to Euler, July 1730)

- Furstenberg's Topological Proof (Furstenberg, 1955)

- Filip Saidak's Proof (Saidak, 2006)

By their sheer definitions alone, namely (i) Gram [y=0] points, which is the usual 'Gram points', being given by $\operatorname{Im}\left\{\zeta\left(\frac{1}{2} \pm\right.\right.$ $t t)\}=0$ and (ii) non-trivial zeros being given by $\zeta\left(\frac{1}{2} \pm t t\right)=0$; it can be seen that they both refer to the same Riemann zeta function specifically at $\sigma=\frac{1}{2}$ value. Respectively, (i) Gram points and (ii) non-trivial zeros refer to this function's generated curve intersecting (i) the $\mathrm{x}$-axis but not the $\mathrm{y}$-axis, thus with $\mathrm{y}=0$ and (ii) both the $\mathrm{x}$-axis and $\mathrm{y}$-axis, thus with $\mathrm{x}=0$ and $\mathrm{y}=0$. That being so, and in concert with this traditional Gram $[\mathrm{y}=0]$ points above, intuitively we can invent the third point of intersection (on the $\mathrm{y}$-axis but not the $\mathrm{x}$-axis) as Gram $[\mathrm{x}=0]$ points characterized by $\operatorname{Re}\left\{\zeta\left(\frac{1}{2} \pm t t\right)\right\}=0$. With Reimann zeta function always generating smooth and continuous curves for all values of $\sigma$, and once we have proven that non-trivial zeros cross over both the $\mathrm{x}$-axis and $\mathrm{y}$-axis with $\mathrm{x}=0$ and $\mathrm{y}=0$ only when $\sigma=\frac{1}{2}$, as was essentially carried out in our previous research paper; then via "proof by association" alone one could arguably concede our proposed Grams conjecture above to be true without going through the rigmarole of doing so from "first principles" as conducted below in this paper.

From 1859 to 2016, the 157 lapsed "years of resistance" before supplying a complete proof for Riemann conjecture / hypothesis may well be an omen that [in stark contrast to the more than one proof possible for 'Prime numbers being infinite in magnitude'] due to the complex nature of Riemann zeta or Dirichlet eta function, there will only be one possible proof ever for this Riemann conjecture / hypothesis. With equal relevance to Gram points, this concept is similarly exploited in the Conclusion section below for our Gram conjecture / hypothesis postulated in 2016.

As the Dirichlet eta function is essentially the surrogate for Riemann zeta function, we treat and closely analyze both functions as unique mathematical objects looking for key intrinsic properties and behaviors. We discovered our key formula (coined the \{Modified-for-Gram points\}-Sigma-power law) and our key Ratio (coined the \{Modified-for-Gram points\}-Riemann-Dirichlet Ratio) with the aid of Dimensional analysis, Ratio study, Calculus, and concepts from the novel Hybrid method of Integer Sequence classification. In so doing we recognize that it is the (i) \{Modified-for-Gram points\}-Sigma-power laws in both the Dirichlet and Riemann versions which are based on either the numerator or denominator part of the (ii) \{Modified-for-Gram points\}-Riemann-Dirichlet Ratio, together with their various underlying mathematically-consistent properties, that crucially provide 'hidden' de novo evidences for the most direct, basic and elementary mathematical proof for Gram conjecture to be true.

The derivation of the two \{Modified-for-Gram points\}-Sigma $(\sigma)$-power laws has a qualitative Dimensional analysis component of equating either the numerator or denominator respective portions from each of the two sub-ratios of the \{Modified-for-Gram points\}-Riemann-Dirichlet Ratio with their underlying complete mathematical expressions. The relevant $\{$ Modified-for-Gram points\}- $\sigma$-power laws derived using either the relevant parameter- $\{2 \mathrm{n}\}$ numerator or shiftedby-one parameter-\{2n-1\} denominator is justifiably mathematically equivalent to each other and also being related by a common proportionality constant. Thus this paper could literally be summed up by the one concise sentence "The Dimensional analysis homogeneity property of the \{Modified-for-Gram points\}-Sigma-power law provides the definitive mathematical proof for Gram conjecture to be true".

\section{Riemann zeta and Dirichlet eta Functions}

Riemann zeta function $\zeta(s)$ at the critical strip is represented by Eq. (1) below where $n=1,2,3, \ldots, \infty$. Instead of the commonly or conventionally used $\mathrm{z}$ symbol for its complex variable, this variable is denoted traditionally in this paper by $s(=\sigma+i t)$ where $i=\sqrt{ }-1$ is the imaginary number; $\sigma$ (consisting of real numbers with values $-\infty<\sigma<+\infty$ ) refers to the argument for the real part of $\mathrm{s}$ [denoted by $\operatorname{Re}\{\mathrm{s}\}]$; and $\mathrm{t}$ (consisting of real numbers with values $-\infty<t<+\infty$ ) refers 
to the argument for the imaginary part of $s$ [denoted by $\operatorname{Im}\{s\}]$.

$$
\begin{aligned}
\zeta(s) & =\frac{1}{\left(1-2^{1-s}\right)} \cdot \sum_{n=1}^{\infty} \frac{(-1)^{n+1}}{n^{s}} \\
& =\frac{1}{1-2^{1-s}} \cdot\left(\frac{1}{1^{s}}-\frac{1}{2^{s}}+\frac{1}{3^{s}}-\cdots\right)
\end{aligned}
$$

Eq. (1) is defined for all $\sigma>0$ except for a simple pole at $\sigma=1$. $\zeta(s)$ without the $\frac{1}{\left(1-2^{1-s}\right)}$ proportionality factor, viz. $\sum_{n=1}^{\infty} \frac{(-1)^{n+1}}{n^{s}}$ is also known as Dirichlet eta $(\eta)$ or alternating zeta function. This $\eta(\mathrm{s})$ function is a holomorphic function of $\mathrm{s}$ as defined by analytic continuation. As $\zeta(s)$ is closely related by the proportionality factor to $\eta(s)$, it can be seen that all Gram points of $\eta(s)$ must be identical to those of $\zeta(s)$ - thus the statement that all the Gram points of $\eta(s)$ in the critical strip are on the critical line $\left(\sigma=\frac{1}{2}\right)$ is in accordance with [an alternative version of] the Gram conjecture is also true.

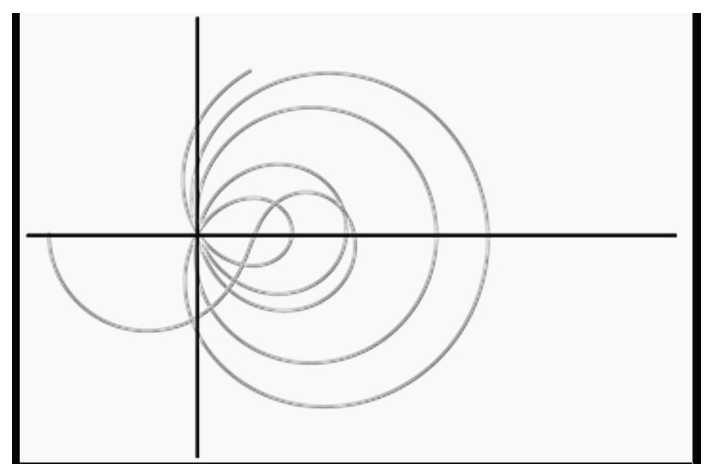

Figure 1a

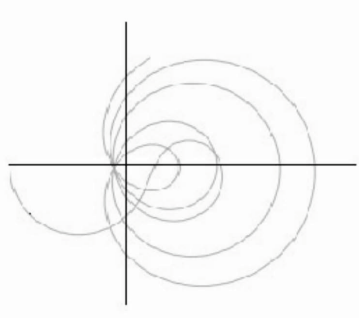

Figure $1 b$

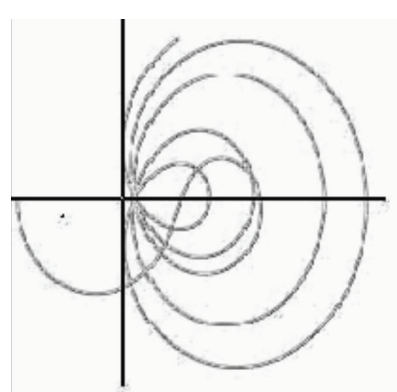

Figure 1c

Schematically depicted polar graph of $\zeta\left(\frac{1}{2}+t t\right)$ in Figure 1a with plot of $\zeta(s)$ along the critical line for real values of $\mathrm{t}$ running from 0 to 34 , horizontal axis: $\operatorname{Re}\left\{\zeta\left(\frac{1}{2}+t t\right)\right\}$, and vertical axis: $\operatorname{Im}\left\{\zeta\left(\frac{1}{2}+t t\right)\right\}$. There is consistent mathematical symmetry about the horizontal axis and a plot of $\zeta\left(\frac{1}{2}-t t\right)$ would have revealed an identical mirror image graph reflected on this axis. Gram points in the critical strip are geometrically visualized as the place where the spirals pass through the horizontal axis (away from the origin). This phenomenon should occur infinitely often as the real number values for $t$ are also infinite. The first 5 non-trivial zeros are seen as the spirals passing through the origin.

Figure $1 \mathrm{~b} \zeta\left(\frac{2}{5}+t t\right)$ on the left and Figure $1 \mathrm{c} \zeta\left(\frac{3}{5}+t t\right)$ on the right are schematically plotted with similar tactics that were used in Figure 1a. Note the relevant spirals with 'opposite' distortion now crossing the horizontal axis along different 'opposing' points to that in Figure 1a. The pictorial representation of the first 5 non-trivial zeros in Figure 1a as the spirals passing through the origin do not occur anymore when the $\sigma$ from $0<\sigma<1$ is of values other than $\frac{1}{2}$. Gram conjecture can also be stated as the condition of total absence of spontaneous occurrence of non-trivial zeros in the critical strip when $\sigma$ satisfy $0<\sigma<\frac{1}{2}$ and $\frac{1}{2}<\sigma<1$ where infinite number of \{Non-critical lines\}-Gram points can be found.

\section{4. \{Modified-for-Gram Points\}-Riemann-Dirichlet Ratio}

Euler formula is commonly stated as $e^{i x}=\cos x+i \cdot \sin x$. The magnificent Euler identity $($ where $x=\pi)$ is $e^{i \pi}=$ $\cos \pi+i \cdot \sin \pi=-1+0$, commonly stated as $e^{i \pi}+1=0$. The $n^{s}$ of Riemann zeta function can be expanded to $n^{s}=$ 
$n^{(\sigma+i t)}=n^{\sigma} \cdot e^{t \cdot \log (n) \cdot i}$ since $n^{t}=e^{t \cdot \log (n)}$. Apply the Euler formula to $n^{s}$ will result in $n^{s}=n^{\sigma} \cdot(\cos (t \cdot \log (n))+i \cdot \sin (t \cdot \log (n))$ - designated here with the short-hand notation $n^{s}$ (Euler) - whereby $n^{\sigma}$ is the modulus and $t \cdot \log (n)$ is the polar angle.

Apply $n^{2}$ (Euler) to Eq. (1), we have $\zeta(s)=\gamma \cdot \eta(s)=\gamma \cdot[\operatorname{Re}\{\eta(s)\}+i \cdot \operatorname{Im}\{\eta(s)\}]$ whereby

$$
\begin{aligned}
& \operatorname{Re}\{\eta(s)\}=\sum_{n=1}^{\infty}\left((2 n-1)^{-\sigma} \cdot \cos (t \cdot \log (2 n-1))-(2 n)^{-\sigma} \cdot \cos (t \cdot \log (2 n))\right) \text { and } \\
& \operatorname{Im}\{\eta(s)\}=i \cdot \sum_{n=1}^{\infty}\left((2 n)^{-\sigma} \cdot \sin (t \cdot \log (2 n))-(2 n-1)^{-\sigma} \cdot \sin (t \cdot \log (2 n))\right)
\end{aligned}
$$

Here $\gamma$ is the proportionality factor $\frac{1}{\left(1-2^{1-s}\right)}$.

As Gram conjecture on Gram points - or more accurately Gram [y=0] points - based on $\zeta(s)$ is identical to that based on its proxy $\eta(s)$, then Gram conjecture - or more accurately Gram [y=0] conjecture - is satisfied when

$$
\sum \operatorname{Re} \operatorname{Im}\{\eta(s)\}=\operatorname{Re}\{\eta(s)\}+0 \text {, or simply } \operatorname{Im}\{\eta(s)\}=0
$$

Applying Eq. (3) to Eq. (2), this equation can be simplified and be reduced to

$$
\begin{aligned}
& \sum_{n=1}^{\infty}\left((2 n)^{-\sigma} \cdot \sin (t \cdot \log (2 n))-(2 n-1)^{-\sigma} \cdot \sin (t \cdot \log (2 n))\right)=0 \\
& \sum_{n=1}^{\infty}(2 n)^{\sigma} \cdot \sin (t \cdot \log (2 n))=\sum_{n=1}^{\infty}(2 n-1)^{-\sigma} \cdot \sin (t \cdot \log (2 n))
\end{aligned}
$$

Eq. (4) completely fulfills the 'presence of the complete set of Gram points' criteria. Rearranging its terms will result in our desired \{Modified-for-Gram points\}-Riemann-Dirichlet Ratio given below.

$$
\frac{\sum_{n=1}^{\infty} \sin (t \cdot \log (2 n))}{\sum_{n=1}^{\infty} \sin (t \cdot \log (2 n-1))}=\frac{\sum_{n=1}^{\infty}(2 n)^{\sigma}}{\sum_{n=1}^{\infty}(2 n-1)^{\sigma}}
$$

Denote the left hand side ratio as Ratio R1 (of a 'cyclical' nature) and the right hand side ratio as Ratio R2 (of a 'noncyclical' nature). Utilizing Ratio study, the \{Modified-for-Gram points\}-Riemann-Dirichlet Ratio can be deemed to be representing a complicated 'dynamic' version of non-Hybrid integer sequence (Ting, 2013) in that besides consisting of identical 'Class function' in each of the two functions when expressed in Ratio R1's numerator and denominator, this first Ratio R1 is again given as an equality to another seemingly different Ratio R2 whose numerator and denominator also consist of identical 'Class function'. Again we recommend the reader to refer to our earlier research article (Ting, 2016) for a fuller explanation of Ratio study, non-Hybrid integer sequence and other associated materials.

The \{Modified-for-Gram points\}-Riemann-Dirichlet Ratio calculations, valid for all continuous real number values of $\mathrm{t}$, would theoretically result in infinitely many non-Hybrid integer sequences [here arbitrarily] for the $0<\sigma<1$ critical strip region of interest with $n=1,2,3, \ldots, \infty$ being discrete integer number values, or $\mathrm{n}$ being continuous real numbers from 1 to $\infty$ with Riemann integral applied in the interval from 1 to $\infty$. This infinitely many integer sequences can geometrically be interpreted to representatively cover the entire plane of the critical strip bounded by $\sigma$ values of 0 and 1 , thus (at least) allowing our proposed proof on Gram conjecture to be of a 'complete' nature.

We apply Riemann integral to the four continuous functions of Ratio R1 and Ratio R2 in Eq. (5) thus depicting the \{Modified-for-Gram points\}-Riemann-Dirichlet Ratio in the integral forms - see the subsequent Eq. (10) in this section and then display Ratio R1 and Ratio R2 physical characteristics for $\sigma=\frac{1}{2}$ at $\mathrm{n}=1$ and $\mathrm{n}=2$ situations in Fig. 2a and Fig. $2 \mathrm{~b}$ respectively below.

Thereafter, step-by-step we derive the closely related \{Modified-for-Gram points\}-Dirichlet $\sigma$-power law [expressed in real numbers] and the \{Modified-for-Gram points\}-Riemann $\sigma$-power law [expressed in real and complex numbers] - these two laws are further elaborated in the following section of this paper. Due to the resemblance to various power-law functions in that the $\sigma$ variable from $s(=\sigma+i t)$ being the exponent of a power function $n^{\sigma}$, the log scale use, and the harmonic $\zeta(s)$ series connection in Zipf's law; we explain here why we have elected to endow our newly derived formula with the name \{Modified-for-Gram points\}-Sigma-power law. Its Dirichlet and Riemann versions are directly related to each other via Dirichlet $\eta(s)$ being the equivalence of Riemann $\zeta(s)$ but without the $\frac{1}{\left(1-2^{1-s}\right)}$ proportionality factor. We stress that it is the main underlying mathematically-consistent properties of symmetry and constraints arising from this power law that also 
allowed our most direct, basic and elementary proof for the Gram conjecture to mature. An important characteristic to note of \{Modified-for-Gram points\}- $\sigma$-power law is that its exact formula expression in the usual mathematical language $\left[y=f\left(x_{1}, x_{2}\right)\right.$ format description for a 2-variable function] consists of $y=\{2 n\}$ or $\{2 n-1\}=f(t, \sigma)$ with $n=1,2,3, \ldots, \infty$ or $\mathrm{n}=1$ to $\infty$ with Riemann integral application; $-\infty<t<+\infty$; and $\sigma$ being of real number values $0<\sigma<1$ corresponding to the [arbitrarily defined] critical strip of interest in this particular case scenario. Individual calculations using \{Modified-for-Gram points\}- $\sigma$-power law for $\sigma=\frac{1}{2}$ and $\sigma=\frac{1}{2} \pm \delta$ (with $\delta$ being a real number between 0 and 0.5) showed an implied mathematical symmetry about the $\sigma=\frac{1}{2}$ value calculation - this is depicted graphically for $\delta=0.3$ in Fig. 3a and Fig. 3b below for \{Modified-for-Gram points\}-Dirichlet $\sigma$-power law expressed in the $\{2 \mathrm{n}\}$ parameter format. For the, initially, $\{2 \mathrm{n}\}$ parameter integration of $\mathrm{R} 1, \int_{1}^{\infty} \sin (t \cdot \log (2 n)) \cdot d n$

Use integration by u-substitution technique to obtain $u=t \cdot \log (2 n), n=\frac{1}{2} e^{\frac{1}{t}(u)}, \frac{d u}{d n}=\frac{2 t}{2 n}=\frac{t}{n}, d u=t \cdot \frac{d n}{n}, d n=2 n \cdot \frac{d u}{2 t}=n \cdot \frac{d u}{t}$ $\int_{1}^{\infty} \sin (u) \cdot \frac{n}{t} \cdot d u=\int_{1}^{\infty} \sin (u) \cdot \frac{1}{t} \cdot \frac{1}{2} \cdot e^{\frac{1}{t}(u)} \cdot d u=\frac{1}{2 t} \int_{1}^{\infty} \sin (u) \cdot e^{\frac{1}{t} u} \cdot d u$

Use the Products of functions proportional to their second derivatives, namely the indefinite integral $\int \sin (a \cdot u) \cdot e^{b \cdot u} d u=$ $\frac{e^{b u}}{a^{2}+b^{2}}(b \cdot \sin (a \cdot u)-a \cdot \cos (a \cdot u))+C$ (Comparatively, we observe that $\left.\int \cos (a \cdot u) \cdot e^{b \cdot u} d u=\frac{e^{b u}}{a^{2}+b^{2}}(b \cdot \cos (a \cdot u)+a \cdot \sin (a \cdot u))+C\right)$. Then $\mathrm{a}=1, \mathrm{~b}=\frac{1}{t}$, and temporarily ignore the $\frac{1}{2 t}$ term, we have

$$
\begin{aligned}
& \int_{1}^{\infty} \sin (u) \cdot e^{\frac{1}{t} u} \cdot d u \\
= & {\left[\left(e^{\frac{1}{t} u}\right) /\left(1+\frac{1}{t^{2}}\right) \cdot\left(\frac{1}{t} \cdot \sin (u)-\cos (u)\right)+C\right]_{1}^{\infty} } \\
= & {\left[\left(t^{2} \cdot e^{\frac{1}{t} u}\right) /\left(t^{2}+1\right) \cdot\left(\frac{1}{t} \cdot \sin (u)-\cos (u)\right)+C\right]_{1}^{\infty} }
\end{aligned}
$$

Now apply the non-linear combination of sine and cosine functions identity, namely $a \cdot \sin (u)+b \cdot \cos (u)=c \cdot \sin (u+\varphi)$ where $c=\sqrt{ }\left(a^{2}+b^{2}\right)$ and $\varphi=a \tan 2(b, a)$. Here $\left.a=\frac{1}{t}, \mathrm{~b}=-1, c=\sqrt{ }\left(\frac{1}{t}\right)^{2}+1\right)=\frac{\sqrt{ }\left(t^{2}+1\right)}{t}$. Then we have

$$
\begin{aligned}
& \int_{1}^{\infty} \sin (u) \cdot e^{\frac{1}{t} u} \cdot d u \\
& =\left[\left(t^{2} \cdot e^{\frac{1}{t} u}\right) /\left(t^{2}+1\right) \cdot \frac{\sqrt{ }\left(t^{2}+1\right)}{t} \cdot \sin (u+\operatorname{atan} 2(b, a))+C\right]_{1}^{\infty} \\
& =\left[\left(t \cdot e^{\frac{1}{t} u}\right) /\left(\sqrt{ }\left(t^{2}+1\right)\right) \cdot \sin (u+\arctan (-t))+C\right]_{1}^{\infty}
\end{aligned}
$$

But there was a $\frac{1}{2 t}$ term in front of this integral as can be seen above. Then after substituting this term and simplifying, the integral

$$
\begin{aligned}
& \int_{1}^{\infty} \sin (u) \cdot e^{\frac{1}{t} u} \cdot d u \\
= & {\left[\left(e^{\frac{1}{t} u}\right) / 2 \sqrt{ }\left(t^{2}+1\right) \cdot \sin (u-\arctan (t))+C\right]_{1}^{\infty} }
\end{aligned}
$$

But $u=t \cdot \log (2 n)$. Reverting back to the $\mathrm{n}$ variable, the equation for the $\{2 \mathrm{n}\}$ parameter finally becomes

$$
\begin{aligned}
& \int_{1}^{\infty} \sin (t \cdot \log (2 n)) \cdot d n \\
& =\left[\left(\{2 n\} \cdot e^{\frac{1}{t}}\right) /\left(2 \sqrt{ }\left(t^{2}+1\right) \cdot \sin (t \cdot \log (2 n)-\arctan (t))+C\right)\right]_{1}^{\infty}
\end{aligned}
$$

In a similar manner integration for the $\{2 \mathrm{n}-1\}$ parameter, this equation becomes

$$
\left[\left(\{2 n-1\} \cdot e^{\frac{1}{t}}\right) /\left(2 \sqrt{ }\left(t^{2}+1\right) \cdot \sin (t \cdot \log (2 n-1)-\arctan (t))+C\right)\right]_{1}^{\infty}
$$

In $\mathrm{R} 2$ using $\{2 \mathrm{n}\}$ parameter,

$$
\begin{aligned}
& \int_{1}^{\infty}(2 n)^{\sigma} d n \\
= & {\left.\left[1 /(2(\sigma+1)) \cdot(2 n)^{\sigma+1}+C\right)\right]_{1}^{\infty} } \\
= & {\left.\left[\frac{1}{3}\{2 n\}(2 n)^{\frac{1}{2}}+C\right)\right]_{1}^{\infty} \text { when } \sigma=\frac{1}{2} }
\end{aligned}
$$


For the equivalent $\mathrm{R} 2$ based on $\{2 n-1\}$ parameter,

$$
\begin{aligned}
& \int_{1}^{\infty}(2 n-1)^{\sigma} d n \\
= & {\left.\left[1 /(2(\sigma+1)) \cdot(2 n-1)^{\sigma+1}+C\right)\right]_{1}^{\infty} } \\
= & {\left.\left[\frac{1}{3}\{2 n-1\}(2 n-1)^{\frac{1}{2}}+C\right)\right]_{1}^{\infty} \text { when } \sigma=\frac{1}{2} }
\end{aligned}
$$

The Ratio R1 and Ratio R2 of \{Modified-for-Gram points\}-Riemann-Dirichlet Ratio (for $\sigma=\frac{1}{2}$ ) is defined by the integral

$$
\frac{\left[\left(\{2 n\} \cdot\left(e^{\frac{1}{t}} \cdot / 2 \sqrt{ }\left(t^{2}+1\right)\right) \cdot \sin (t \cdot \log (2 n)-\arctan (t))\right]_{1}^{\infty}\right.}{\left[\left(\{2 n-1\} \cdot e^{\frac{1}{t}} / 2 \sqrt{ }\left(t^{2}+1\right)\right) \cdot \sin (t \cdot \log (2 n-1)-\arctan (t))\right]_{1}^{\infty}}=\frac{\left[\frac{1}{3}\{2 n\}(2 n)^{\frac{1}{2}}\right]_{1}^{\infty}}{\left[\frac{1}{3}\{2 n-1\}(2 n-1)^{\frac{1}{2}}\right]_{1}^{\infty}}
$$

Cancelling out the common parameter $\{2 \mathrm{n}\}$ and $\{2 \mathrm{n}-1\}$ terms,

$$
\begin{aligned}
& \frac{\left[\left(e^{\frac{1}{t}} / 2 \sqrt{ }\left(t^{2}+1\right)\right) \cdot \sin (t \cdot \log (2 n)-\arctan (t))\right]_{1}^{\infty}}{\left[\left(e^{\frac{1}{t}} / 2 \sqrt{ }\left(t^{2}+1\right)\right) \cdot \sin (t \cdot \log (2 n-1)-\arctan (t))\right]_{1}^{\infty}} \leftarrow \text { this is R1 } \\
= & \frac{\left[\frac{1}{3}(2 n)^{\frac{1}{2}}\right]_{1}^{\infty}}{\left[\frac{1}{3}(2 n-1)^{\frac{1}{2}}\right]_{1}^{\infty}} \leftarrow \text { this is R2 }
\end{aligned}
$$

Figure 2a. \{Modified-for-Gram points\}-Riemann-Dirichlet Ratio displayed as Ratio R1 and Ratio R2 for $\sigma=\frac{1}{2}$ at $\mathrm{n}=$

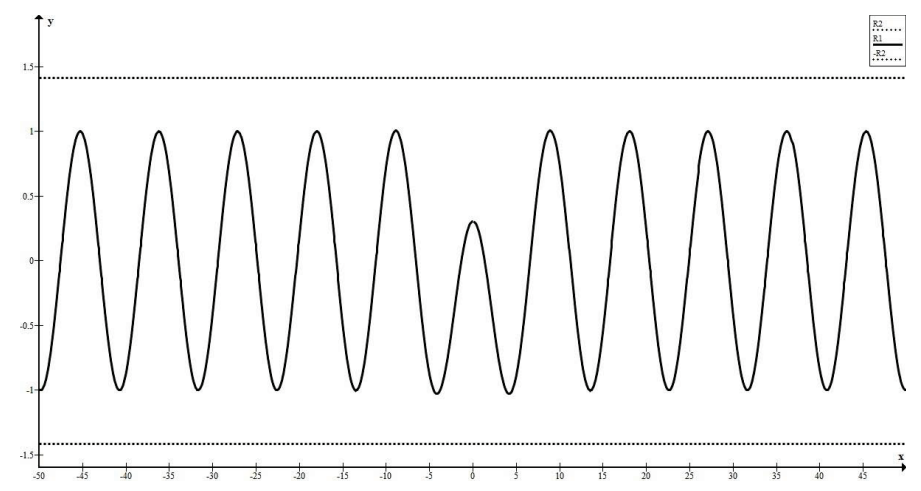

Figure 2a for Gram $[\mathrm{y}=0]$ points

1 situation. $\mathrm{x}$-axis $=\mathrm{t}, \mathrm{y}$-axis $=\mathrm{R} 1$ and $\mathrm{R} 2$ values. Note $\mathrm{R} 2$ lines seem to mathematically constraint the maxima and minima values of $\mathrm{R} 1$.

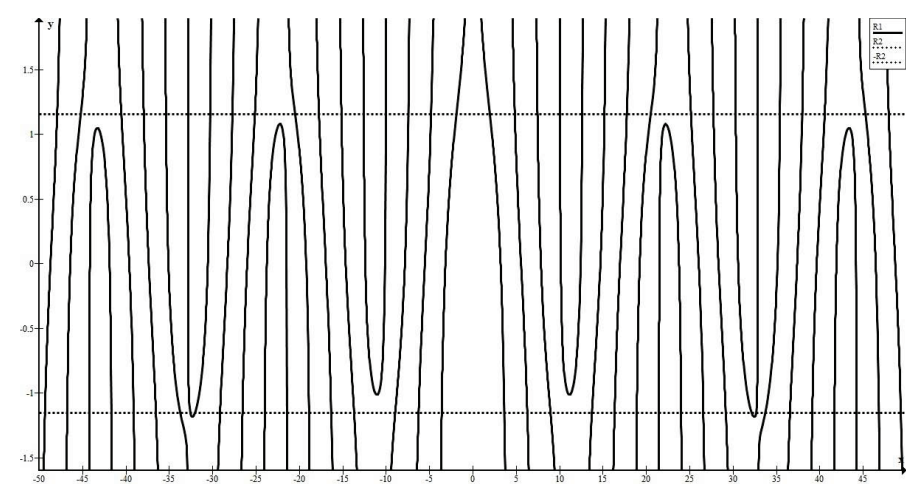

Figure $2 \mathrm{~b}$ for Gram $[\mathrm{y}=0]$ points

Figure 2b. \{Modified-for-Gram points\}-Riemann-Dirichlet Ratio displayed as Ratio R1 and Ratio R2 for $\sigma=\frac{1}{2}$ at $\mathrm{n}=2$ situation. $\mathrm{x}$-axis $=\mathrm{t}, \mathrm{y}$-axis $=\mathrm{R} 1$ and $\mathrm{R} 2$ values. The $\mathrm{R} 2$ lines seem to mathematically constraint the maxima and minima values of $\mathrm{R} 1$ but $\mathrm{R} 1$ overall sinusoidal landscape is now vastly different to that of Figure $2 \mathrm{a}$ - we note here that $\mathrm{n}$ goes from $1,2,3, \ldots, \infty$ and each $\mathrm{n}$ value could be graphically represented manifesting Fractals with self-similarity. 


\section{5. \{Modified-for-Gram Points\}-Sigma-power Law}

The $\gamma$ proportionality factor term in Riemann $\zeta$ function, viz. $\frac{1}{\left(1-2^{1-s}\right)}$, can also be expressed with the aid of Euler formula as follows (with the formula for $\sigma=\frac{1}{2}$ substitution depicted last).

$$
\begin{aligned}
& \frac{1}{\left(1-2^{1-s}\right)} \\
= & \left(2^{\sigma} \cdot 2^{i t}\right) /\left(2^{\sigma} \cdot 2^{i t}-2\right) \\
= & \left(2^{\sigma} \cdot e^{t \cdot \log (2 i)}\right) /\left(2^{\sigma} \cdot e^{t \cdot \log (2 i)}-2\right) \\
= & \left(2 ^ { \sigma } \cdot \left(\operatorname { c o s } \left(t \cdot \log (2)+i \cdot \sin (t \cdot \log (2)) /\left(2^{\sigma} \cdot(\cos (t \cdot \log (2)+i \cdot \sin (t \cdot \log (2)-2)\right.\right.\right.\right. \\
= & \left(2 ^ { \frac { 1 } { 2 } } \cdot \left(\operatorname { c o s } \left(t \cdot \log (2)+i \cdot \sin (t \cdot \log (2)) /\left(2^{\frac{1}{2}} \cdot(\cos (t \cdot \log (2)+i \cdot \sin (t \cdot \log (2)-2)\right.\right.\right.\right.
\end{aligned}
$$

The \{Modified-for-Gram points\}-Dirichlet and \{Modified-for-Gram points\}-Riemann $\sigma$-power laws are given by the exact formulae in Eqs. (12) to (15) below with $\psi$ being the same proportionality constant valid for both power laws. We can now dispense with the constant of integration C. Using Dimensional analysis approach we can easily conclude that the 'fundamental dimension' [Variable / Parameter / Number X to the power of Number Y] has to be represented by the particular 'unit of measure' [Variable / Parameter / Number $X$ to the power of Number $Y$ whereby Number $\mathrm{Y}$ needs to be of the specific value $\frac{1}{2}$ ] for Dimensional analysis homogeneity to occur. This de novo Dimensional analysis homogeneity equates to the location of the complete set of Gram points and is crucially a fundamental property present in all laws of Physics. The 'unknown' $\sigma$ variable, now endowed with the value of $\frac{1}{2}$, is treated as Number Y.

\{Modified-for-Gram points $\}$-Dirichlet $\sigma$-power law using the $\{2 \mathrm{n}\}$ parameter:

$$
\left.\left[\{2 n\} \cdot \frac{e^{\frac{1}{t}}}{2\left(t^{2}+1\right)^{\frac{1}{2}}} \cdot \sin (t \cdot \log (2 n)-\arctan (t))\right]_{1}^{\infty}=\psi \cdot \frac{1}{3}\{2 n\}(2 n)^{\frac{1}{2}}\right]_{1}^{\infty}
$$

With the common parameter $\{2 \mathrm{n}\}$ cancelling out on both sides, the equation reduces to

$$
\left[\frac{e^{\frac{1}{t}}}{2\left(t^{2}+1\right)^{\frac{1}{2}}} \cdot \sin (t \cdot \log (2 n)-\arctan (t))-\psi \cdot \frac{1}{3}(2 n)^{\frac{1}{2}}\right]_{1}^{\infty}=0
$$

Similarly for the $\{2 \mathrm{n}-1\}$ parameter, this equivalent equation is

$$
\left[\frac{e^{\frac{1}{t}}}{2\left(t^{2}+1\right)^{\frac{1}{2}}} \cdot \sin (t \cdot \log (2 n-1)-\arctan (t))-\psi \cdot \frac{1}{3}(2 n-1)^{\frac{1}{2}}\right]_{1}^{\infty}=0
$$

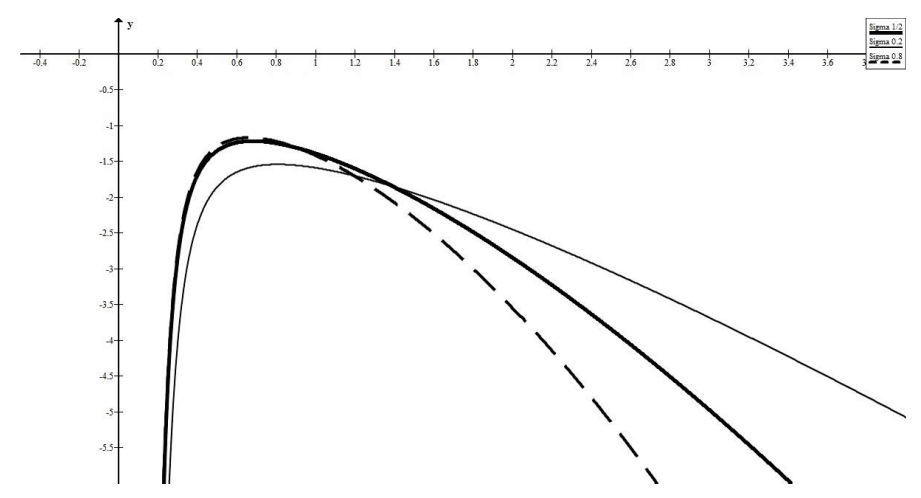

Figure $3 \mathrm{a}$ for Gram $[\mathrm{y}=0]$ points

Figure 3a. \{Modified-for-Gram points\}-Dirichlet $\sigma$-power law: using $\{2 \mathrm{n}\}$ parameter, $\mathrm{n}=1$ situation, $\mathrm{x}$-axis $=\mathrm{t}, \psi$ arbitrarily defined with value $1, \mathrm{y}$-axis $=\sigma$-power law values obtained when $\sigma=0.2$ (non-critical line), $\frac{1}{2}$ (critical line where all Gram points lie), and 0.8 (non-critical line) displayed on the usual linear-linear graph.

Figure 3b. \{Modified-for-Gram points\}-Dirichlet $\sigma$-power law: using the same criteria and data to that obtained in Figure 3a except that they are now displayed on the log-linear graph, with log here referring to logarithm in base 10 . Note (i) 


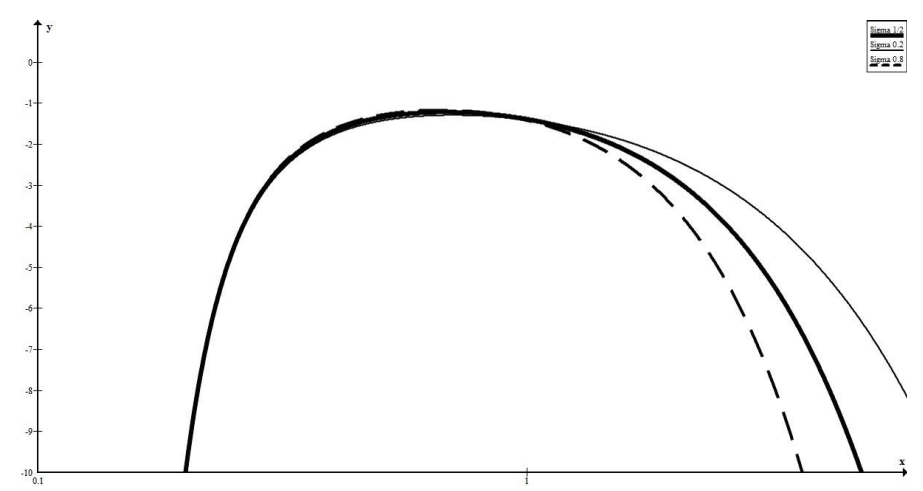

Figure $3 \mathrm{~b}$ for Gram $[\mathrm{y}=0]$ points

the mathematical requirement of the y-axis in Figure $3 b$ to be linear [and not with log scale] due to the negative values obtained for y-axis and (ii) the implied mathematical symmetry about the $\sigma=\frac{1}{2}$ value in both Figure 3a and Figure $3 \mathrm{~b}$.

Finally, the \{Modified-for-Gram points\}-Riemann $\sigma$-power law is given by the exact formulae using $\{2 \mathrm{n}\}$ and $\{2 \mathrm{n}-1\}$ parameters with the $\gamma=\frac{2^{\frac{1}{2}} \cdot(\cos (t \cdot \log (2)+i \cdot \sin (t \cdot \log (2))}{\left(2^{\frac{1}{2}} \cdot(\cos (t \cdot \log (2)+i \cdot \sin (t \cdot \log (2)-2)\right.}$ substitution.

$$
\begin{aligned}
& {\left[\frac{e^{\frac{1}{t}}}{2\left(t^{2}+1\right)^{\frac{1}{2}}} \cdot \sin (t \cdot \log (2 n)-\arctan (t))-\psi \cdot \gamma \cdot \frac{1}{3} \cdot(2 n)^{\frac{1}{2}}\right]_{1}^{\infty}=0} \\
& {\left[\frac{e^{\frac{1}{t}}}{2\left(t^{2}+1\right)^{\frac{1}{2}}} \cdot \sin (t \cdot \log (2 n)-\arctan (t))-\psi \cdot \frac{2^{\frac{1}{2}} \cdot(\cos (t \cdot \log (2)+i \cdot \sin (t \cdot \log (2)))}{2^{\frac{1}{2}} \cdot(\cos (t \cdot \log (2)+i \cdot \sin (t \cdot \log (2))-2)} \cdot \frac{1}{3} \cdot(2 n)^{\frac{1}{2}}\right]_{1}^{\infty}=0} \\
& {\left[\frac{e^{\frac{1}{t}}}{2\left(t^{2}+1\right)^{\frac{1}{2}}} \cdot \sin (t \cdot \log (2 n-1)-\arctan (t))-\psi \cdot \gamma \cdot \frac{1}{3} \cdot(2 n-1)^{\frac{1}{2}}\right]_{1}^{\infty}=0} \\
& {\left[\frac{e^{\frac{1}{t}}}{2\left(t^{2}+1\right)^{\frac{1}{2}}} \cdot \sin (t \cdot \log (2 n-1)-\arctan (t))-\psi \cdot \frac{2^{\frac{1}{2}} \cdot(\cos (t \cdot \log (2)+i \cdot \sin (t \cdot \log (2)))}{2^{\frac{1}{2}} \cdot(\cos (t \cdot \log (2)+i \cdot \sin (t \cdot \log (2))-2)} \cdot \frac{1}{3} \cdot(2 n-1)^{\frac{1}{2}}\right]_{1}^{\infty}=0}
\end{aligned}
$$

Note the $\gamma$ proportionality factor given by Eq. (11) above when depicted with the $2^{\frac{1}{2}}$ constant numerical value (derived using $\sigma=\frac{1}{2}$ as proposed in the original Gram conjecture) further allowing, and enabling, de novo Dimensional analysis homogeneity compliance in \{Modified-for-Gram points\}-Riemann $\sigma$-power law.

We illustrate the Dimensional analysis non-homogeneity property for an $\sigma=\frac{1}{4}$ arbitrarily chosen value [clear-cut case with $\{2 \mathrm{n}\}$-parameter] of \{Modified-for-Gram points\}-Riemann $\sigma$-power law lying on a non-critical line (with total absence of Gram points) in the following formula derived using Eq. (11) and Eq. (15). As Ratio R1 component of \{Modified-forGram points\}-Riemann-Dirichlet Ratio is independent of $\sigma$ variable, unlike the Ratio R2 component of \{Modifiedfor-Gram points\}-Riemann-Dirichlet Ratio and the $\gamma$ proportionality factor which are dependent on $\sigma$ variable, we now note the mixture of $\frac{1}{4}$ and $\frac{1}{2}$ exponents subtly, but nevertheless, present in this formula confirming Dimensional analysis non-homogeneity. Also the replacement of $\frac{1}{3}$ fraction with $\frac{2}{5}$ fraction [derived from substituting $\sigma=\frac{1}{4}$ into $\left.\frac{1}{2(\sigma+1)}\right]$ has occurred. Mathematically, this Dimensional analysis non-homogeneity property for any real number value of $\sigma$, when $\sigma \neq \frac{1}{2}$ and $0<\sigma<1$, will always be present indicative of the full presence of \{Non-critical lines\}-Gram points, or by the same token, indicative of total absence of Gram points.

$$
\left[\frac{e^{\frac{1}{t}}}{2\left(t^{2}+1\right)^{\frac{1}{2}}} \cdot \sin (t \cdot \log (2 n)-\arctan (t))-\psi \cdot \frac{2^{\frac{1}{4}} \cdot(\cos (t \cdot \log (2)+i \cdot \sin (t \cdot \log (2)))}{2^{\frac{1}{4}} \cdot(\cos (t \cdot \log (2)+i \cdot \sin (t \cdot \log (2))-2)} \cdot \frac{2}{5} \cdot(2 n)^{\frac{1}{4}}\right]_{1}^{\infty}=0
$$

The $\frac{1}{2}$ exponent in Eq. (16) only occur once in the denominator of the first term. The subtlety of Dimensional analysis nonhomogeneity for \{Non-critical lines\}-Gram points is even more pronounced when compared to its closely related cousin equation (obtained from our previous paper (Ting, 2016)) for Riemann $\sigma$-power law [given below for easy clarification and confirmation with the $\frac{1}{2}$ exponent occurring twice in the first term].

$$
\left[2^{\frac{1}{2}} \cdot \frac{e^{\frac{1}{t} \cdot \frac{3}{4} \pi}}{2\left(t^{2}+1\right)^{\frac{1}{2}} \cdot e^{\frac{3}{4} \pi}} \cdot \sin \left(t \cdot \log (2 n)+\frac{3}{4} \pi-\arctan (t)\right)-\psi \cdot \frac{2^{\frac{1}{4}} \cdot(\cos (t \cdot \log (2)+t \cdot \sin (t \cdot \log (2)))}{2^{\frac{1}{4}} \cdot(\cos (t \cdot \log (2)+t \cdot \sin (t \cdot \log (2))-2))} \cdot \frac{2}{5}(2 n)^{\frac{1}{4}}\right]_{1}^{\infty}=0
$$




\section{Conclusions}

The reader will unmistakenly notice our intentional extremely parallel depiction of written materials between the current and our previous research article (Ting, 2016) concentrating (respectively) on Gram points and non-trivial zeros. After all, Gram points - or more accurately Gram $[\mathrm{y}=0]$ points - and non-trivial zeros can both be regarded as different facets of the roots (or zeros) of the same Riemann zeta function at the same critical line manifesting as "roots at $\mathrm{x}$-axis with $\mathrm{y}$ $=0$ " and "roots at both $\mathrm{x}$ - and $\mathrm{y}$-axis with $\mathrm{x}=0$ and $\mathrm{y}=0$ " (respectively). Then Gram $[\mathrm{x}=0]$ points obviously refer to "roots at $\mathrm{y}$-axis with $\mathrm{x}=0$ ". Note that non-trivial zeros can also be more accurately denoted by Gram $[\mathrm{x}=0, \mathrm{y}=0]$ points. The seemingly small but utterly essential mathematical step in recognizing and representing a 2-variable function with parameters $\{2 \mathrm{n}\}$ or $\{2 \mathrm{n}-1\}$ allows crucial moments where cancellation of the relevant "common" parameters in \{Modifiedfor-Gram points\}-Riemann-Dirichlet Ratio and various \{Modified-for-Gram points\}-Sigma-power laws can occur, further allowing the proper Dimensional analysis process to happen in the absolute correct way. These "common" parameters must be mathematically viewed as $(2 n)^{1}$ or $(2 n-1)^{1}$, viz. raised to a power (exponent) of 1 which will hamper proper Dimensional analysis if not serendipitously deleted - contrast this scenario with the presence of parameters $(2 n)^{\frac{1}{2}}$ or $(2 n-1)^{\frac{1}{2}}$, viz. raised to a power (exponent) of $\frac{1}{2}$ which will then enable proper Dimensional analysis (homogeneity) to proceed. The mathematical foot-prints (6 steps) of this paper are: Step 1: Riemann zeta or Dirichlet eta function [for the critical strip $0<\sigma<1$ ] $\rightarrow$ Step 2: Riemann zeta or Dirichlet eta function [with Euler formula application] $\rightarrow$ Step 3: Riemann zeta or Dirichlet eta function [simplified and identical version specifically indicating the criteria for the presence of the complete set of Gram points] $\rightarrow$ Step 4: \{Modified-for-Gram points\}-Riemann-Dirichlet Ratio [in discrete summation format] $\rightarrow$ Step 5: \{Modified-for-Gram points\}-Riemann-Dirichlet Ratio [in continuous integral format] $\rightarrow$ Step 6: \{Modified-for-Gram points\}-Riemann Sigma-power law and \{Modified-for-Gram points\}-Dirichlet Sigma-power law [both with Dimensional analysis homogeneity].

Note that \{Modified-for-Gram points\}-Riemann Sigma-power law and \{Modified-for-Gram points\}-Dirichlet Sigma-power law, both with Dimensional analysis homogeneity indicative of the critical line when $\sigma=\frac{1}{2}$, coincide with the presence of the complete set of Gram points whereas \{Modified-for-Gram points\}-Riemann Sigma-power law and \{Modified-for-Gram points\}-Dirichlet Sigma-power law, both with Dimensional analysis non-homogeneity indicative of the infinite number of non-critical lines when $0<\sigma<\frac{1}{2}$ and $\frac{1}{2}<\sigma<1$, coincide with the total absence of Gram points [or equivalently stated as coincide with the total presence of complete set of \{Non-critical lines\}-Gram points].

Ratio study of the \{Modified-for-Gram points\}-Riemann-Dirichlet Ratio indicated that it is directly derived from, and related to, either Riemann zeta or Dirichlet eta function via mathematically rearranging their respective 'Euler formulaenriched' infinite series, and then further transforming the original Ratio's 'discrete summation' format into the 'continuous integration' format using Riemann integral. This Ratio crucially incorporated the criteria for the presence of all (identical) Gram points for Riemann zeta function [and Dirichlet eta function by default] in a de novo manner. Both the \{Modified-for-Gram points\}-Riemann and \{Modified-for-Gram points\}-Dirichlet Sigma power-laws are simply derivations of either the numerator or denominator portion of \{Modified-for-Gram points\}-Riemann-Dirichlet Ratio.

The completeness of our proof is fully supported by the fact that the critical strip $(0<\sigma<1)$ is fully represented by the one and only one critical line $\left(\sigma=\frac{1}{2}\right)$ in the middle and an infinite number of other parallel lines $(0<\sigma<1$ and $\sigma \neq \frac{1}{2}$ ) on either side of this critical line, with every single line mathematically described by Riemann zeta function with a particular designated $\sigma$ value. The Dimensional analysis homogeneity property of \{Modified-for-Gram points\}Riemann and \{Modified-for-Gram points\}-Dirichlet Sigma-power laws provides the definitive mathematical proof for Gram conjecture to be true - stated in this manner, this statement also "coincides" exactly with the critical line $\left(\sigma=\frac{1}{2}\right)$ location of all Gram points [as conjectured by the original Gram conjecture]. All the other infinite numbers of non-critical lines have Dimensional analysis with non-homogeneity property, and thus will not contain Gram points. In Dimensional analysis language, there is one, and only one, 'valid' equation at $\sigma=\frac{1}{2}$ complying with Dimensional analysis homogeneity property. Likewise, all the non-critical lines consist of Dimensional analysis 'invalid' equations as they fail to comply with Dimensional analysis homogeneity property. It is also a non-negotiable scientific principle that compliance and mathematical constraint of physical laws and mathematical equations with Dimensional analysis homogeneity enables fundamental laws of Physics and Mathematics to be correct.

The synopsis in the previous four paragraphs broadly summarizes the proposed findings of this study as outlined in the above sections of this paper, in particular via Theorem I to IV from our Introduction section. We theorized that with the correctness of those four theorems, although deceptively simplistic in nature, this fait accompli will now respectfully supply the complete proof for Gram conjecture at the most fundamental level - this could also equivalently be stated as peer review's inability to falsify those four theorems. It is noted that neither the \{Modified-for-Gram points\}-RiemannDirichlet Ratio nor the two \{Modified-for-Gram points\}-Sigma-power laws by themselves have the means to be able to calculate the precise location of each Gram point. However, this is not at all essential for the purpose of our study. 
There is one, and only one, exact mathematical equation of Riemann zeta function able to validly represent the critical strip, viz. $\zeta(s)=\operatorname{Re}\{\zeta(s)\}+\operatorname{l} \cdot \operatorname{Im}\{\zeta(s)\}$ - its real and imaginary components derived from $\frac{1}{\left(1-2^{1-s}\right)} \cdot \sum_{n=1}^{\infty} \frac{(-1)^{n+1}}{n^{s}}$, with implied Gram points 'location' signature capability able to be incorporated into its equivalently derived and expanded equation(s). One sense here that, intuitively, the original Formula $\mathrm{X}$ that does enable calculations for the precise locations of Gram points, by itself, will be totally resistant to being used alone [if at all] in actually proving our Gram conjecture. Likely the added-on complexity afforded by the alternating positive and negative terms of Riemann zeta and Dirichlet eta functions, as a 'weak' principle, totally preclude this from happening. By aesthetic [but incorrect] reasoning alone for this case, this may simply be because the calculated values for the precise locations of particular Gram points per se from Formula $\mathrm{X}$ does not in principle guarantee that there is not another mathematically 'different' Formula $\mathrm{Y}$ out there that will also enable calculations for the precise locations of some other 'extra' Gram points located away from the critical line. Philosophically, we tantalized with a 'strong' principle that our Ratio study approach may well be the only option or 'lone' method to use when attempting to solve our proposed Gram conjecture problem.

Ultimately the direct mathematical connection between the criteria for the presence of Gram points in Riemann zeta (or Dirichlet eta) function with the Dimensional analysis homogeneity criteria in \{Modified-for-Gram points\}-Sigma-power laws, all occurring at the one $\sigma=\frac{1}{2}$ value, is in complete favor for our general argument on Gram conjecture to be true. This is simply because the solution for $\sigma$ variable to be $\frac{1}{2}$ is to enable full compliance with Dimensional analysis homogeneity for \{Modified-for-Gram points\}-Riemann Sigma-power law and \{Modified-for-Gram points\}-Dirichlet Sigma-power law - now seen as Dimensional analysis-wise truly valid mathematical equations or physical laws [in Step 6 of our mathematical foot-prints] - is undeniably also the effective correct solution to Riemann zeta and Dirichlet eta function as simplified and identical versions specifically indicating the criteria for the presence of the complete set of Gram points [in Step 3 of our mathematical foot-prints]

Two refined explanations for Paragraph 1 above are (i) Our perceived mathematical preference to perform Dimensional analysis on \{Modified-for-Gram points\}-Sigma-power laws instead of \{Modified-for-Gram points\}-Riemann-Dirichlet Ratio is because the former is more succinct in that it involves "only one solitary parameter $\{2 \mathrm{n}\}$ or $\{2 \mathrm{n}-1\}$ in equation format" while the later involves "both parameters $\{2 n\}$ and $\{2 n-1\}$ in ratio format", and (ii) There is a precise [interpretational] complete association between non-trivial zeros and Gram points both lying on the same critical line in that the infinite presence of one absolutely guarantee the infinite presence of the other.

Our respectful final comment and opinion is that the proposed Gram conjecture has justifiably become Gram hypothesis which refers to the rigorously proven mathematical statement that all Gram points only occur on the critical line $\left[\sigma=\frac{1}{2}\right]$ in the critical strip $[0<\sigma<1]$ of Riemann zeta function when the unconditional scientific principle of Dimensional analysis homogeneity is not violated. Thus this Gram hypothesis statement is in essence the "quod erat demonstrandum(Q.E.D)-equivalent" proof for our Gram conjecture.

Dirichlet-Gram-Riemann conjecture is the mathematical postulate that all of the Gram $[x=0]$ points, Gram $[y=0]$ points, and Gram $[x=0, y=0]$ points only occur on the critical line $\left[\sigma=\frac{1}{2}\right]$ in the critical strip $[0<\sigma<1]$ of Riemann zeta function when the unconditional scientific principle of Dimensional analysis homogeneity is not violated.

(I) Jørgen Pedersen Gram (June 27, 1850 - April 29, 1916): Danish mathematician. (II) Johann Peter Gustav Lejeune Dirichlet (February 13, 1805 - May 5, 1859): German mathematician. (III) Georg Friedrich Bernhard Riemann (September 17, 1826 - July 20, 1866): German mathematician.

Finally as honor and tribute to the three namesake famous mathematicians, we devise the glorified version Dirichlet-GramRiemann conjecture above by simply combining the individual proofs for Gram $[\mathrm{x}=0]$ points, Gram [y=0] points, and Gram $[\mathrm{x}=0, \mathrm{y}=0]$ points to require that $\sigma$ have the $\frac{1}{2}$ value in order for all of them to co-exist. Here we reiterate again that Gram $[y=0]$ and Gram $[x=0, y=0]$ are simply the usual/traditional Gram points and non-trivial zeros (respectively). This global conjecture can be seen to be proven and become the thoroughly established Dirichlet-Gram-Riemann hypothesis - which is the rigorously proven mathematical statement that all Gram $[x=0]$ points, Gram $[y=0]$ points, and Gram $[\mathbf{x}=\mathbf{0}, \mathbf{y}=\mathbf{0}]$ points only occur on the critical line $\left[\sigma=\frac{1}{2}\right]$ in the critical strip $[0<\sigma<1]$ of Riemann zeta function when the unconditional scientific principle of Dimensional analysis homogeneity is not violated - once we succintly summarize the finer points for the proof associated with the case of Gram $[\mathrm{x}=0]$ points. The equivalent of Fig. 2a, Fig. 2b, Fig. 3a, and Fig. 3b are respectively depicted below by Fig. 4a, Fig. 4b, Fig. 5a, and Fig. 5b for the Gram [x=0] points situation. For Gram [x=0] points, Gram [x=0] conjecture is satisfied by Eq. (18) below, whereby Eq. (18) is the equivalent of Eq. (3) above.

$$
\sum \operatorname{Re} \operatorname{Im}\{\eta(s)\}=0+\operatorname{Im}\{\eta(s)\} \text {, or simply } \operatorname{Re}\{\eta(s)\}=0
$$




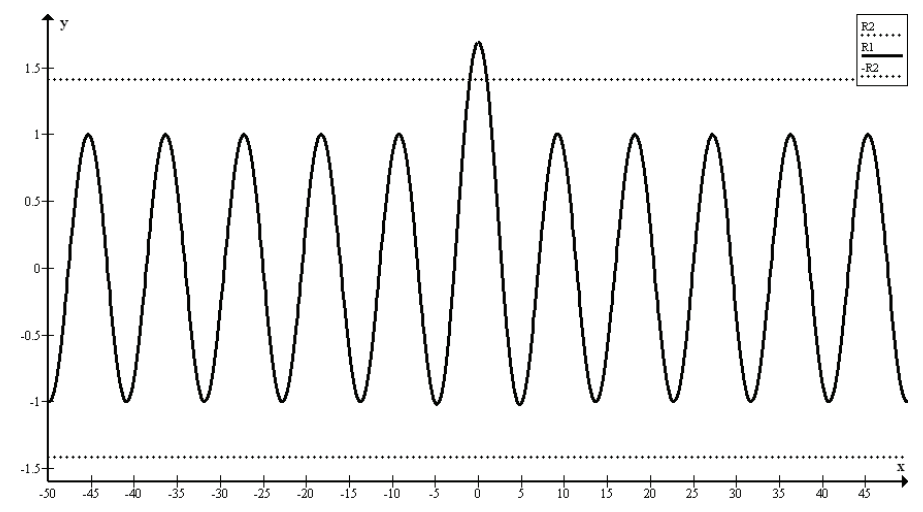

Figure 4a for Gram $[\mathrm{x}=0]$ points

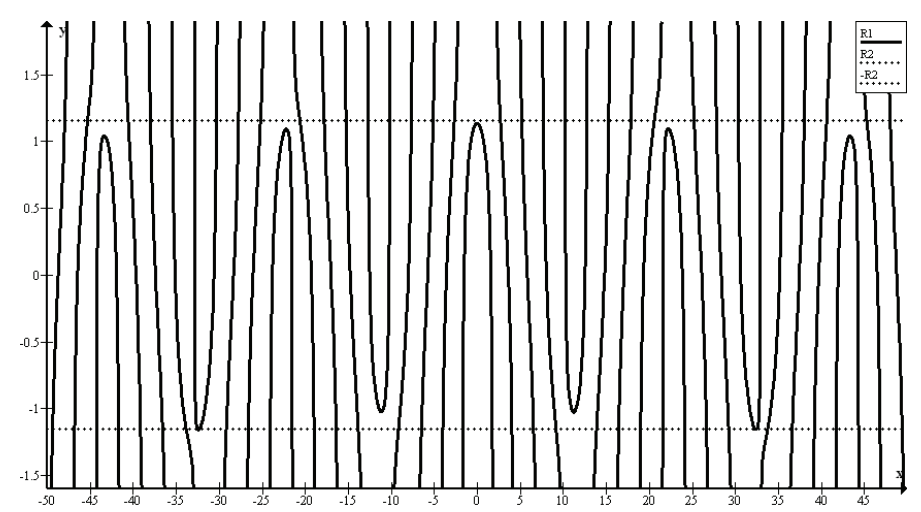

Figure $4 \mathrm{~b}$ for Gram $[\mathrm{x}=0]$ points

Not unexpectedly with only minor subtraction (-) operator to addition (+) operator sign change required, the equivalent to Eq. (14) and Eq. (16) above using $\{2 \mathrm{n}\}$ parameter for Gram $[\mathrm{x}=0]$ points can easily be derived to (respectively) be:

$$
\begin{gathered}
{\left[\frac{e^{\frac{1}{t}}}{2\left(t^{2}+1\right)^{\frac{1}{2}}} \cdot \sin (t \cdot \log (2 n)+\arctan (t))-\psi \cdot \frac{2^{\frac{1}{2}} \cdot(\cos (t \cdot \log (2)+i \cdot \sin (t \cdot \log (2)))}{2^{\frac{1}{2}} \cdot(\cos (t \cdot \log (2)+i \cdot \sin (t \cdot \log (2))-2)} \cdot \frac{1}{3} \cdot(2 n)^{\frac{1}{2}}\right]_{1}^{\infty}=0} \\
{\left[\frac{e^{\frac{1}{t}}}{2\left(t^{2}+1\right)^{\frac{1}{2}}} \cdot \sin (t \cdot \log (2 n)+\arctan (t))-\psi \cdot \frac{2^{\frac{1}{4}} \cdot(\cos (t \cdot \log (2)+i \cdot \sin (t \cdot \log (2)))}{2^{\frac{1}{4}} \cdot(\cos (t \cdot \log (2)+i \cdot \sin (t \cdot \log (2))-2)} \cdot \frac{2}{5} \cdot(2 n)^{\frac{1}{4}}\right]_{1}^{\infty}=0}
\end{gathered}
$$

Dimensional analysis homogeneity and non-homogeneity are demonstrated once again by Eq. 19 and Eq. 20 respectively. 


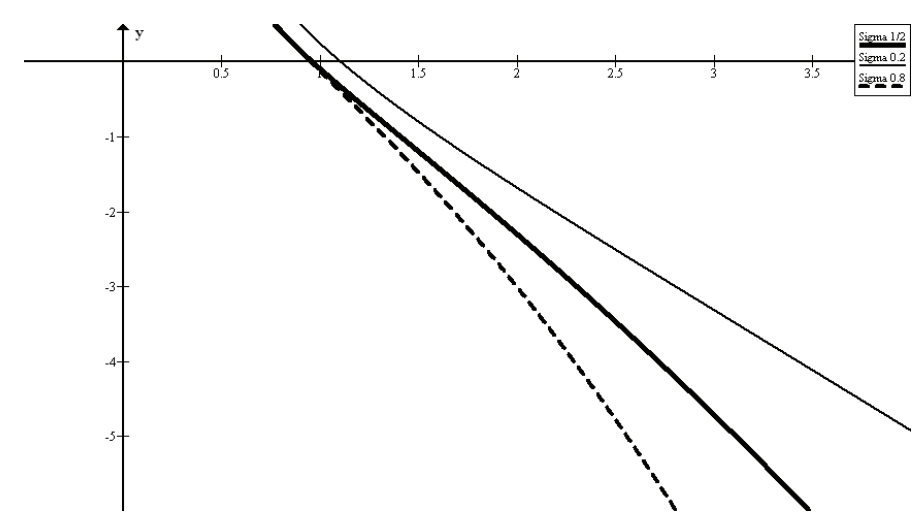

Figure 5a for Gram $[\mathrm{x}=0]$ points

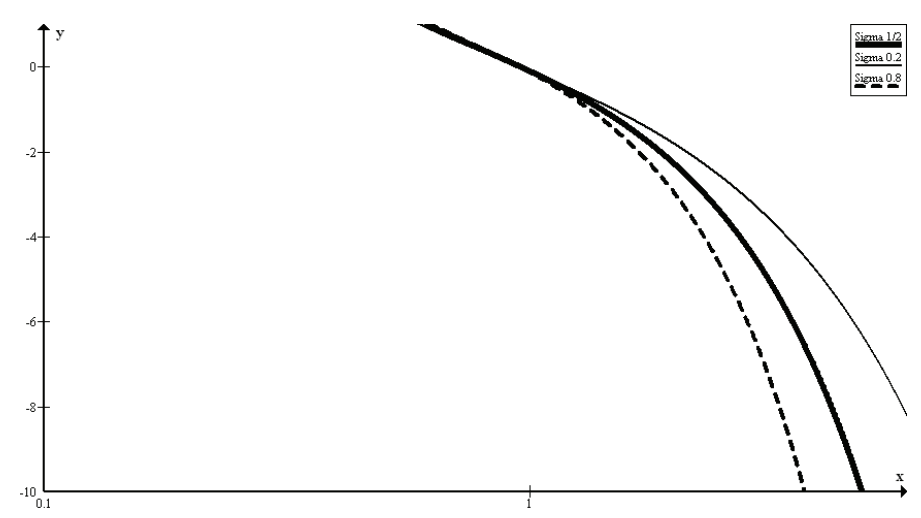

Figure $5 \mathrm{~b}$ for Gram $[\mathrm{x}=0]$ points

\section{Acknowledgements}

The author is indebted to Mr. Rodney Williams (with dual Engineer \& Mathematics degree qualifications from Australia) for his constructive criticism, and the reviewers (participating in the double blind peer-review) for their helpful feedback, on this paper. To the loving memory of Jasmine (and Grace) who had provided deep inspirations to many in 2015 (and 2016) and was a caring auntie (and grandmother) to Jelena, the author's 27-weeker premature daughter born in 2012.

\section{References}

Furstenberg, H. (1955). On the infinitude of primes. Amer. Math. Monthly, 62(5), 353. http://dx.doi.org/10.2307/2307043

Korolev, M. (2012). Gram's law and the argument of the Riemann zeta function. Publications de l'Institut Mathematique, 92(106), 53. http://dx.doi.org/10.2298/PIM1206053K

Saidak, F. (2006), A New Proof of Euclid's theorem, Amer. Math. Monthly, 113(10), 937. http://dx.doi.org/10.2307/27642094

Ting, J. (2013). A228186. The On-line Encyclopedia of Integer Sequences. https://oeis.org/A228186

Ting, J. Y. C. (2016). Rigorous proof for Riemann hypothesis using the novel Sigma-power laws and concepts from the Hybrid method of Integer Sequence classification. J. Math. Res. 8(3), 9. http://dx.doi.org/10.5539/jmr.v8n3p9

\section{Copyrights}

Copyright for this article is retained by the author(s), with first publication rights granted to the journal.

This is an open-access article distributed under the terms and conditions of the Creative Commons Attribution license (http://creativecommons.org/licenses/by/3.0/). 